\title{
Folate receptor targeting of radiolabeled liposomes reduces intratumoral liposome accumulation in human KB carcinoma xenografts
}

This article was published in the following Dove Press journal: International Journal of Nanomedicine

\author{
Esben Christensen ${ }^{1-3}$ \\ Jonas R Henriksen ${ }^{2,4}$ \\ Jesper T Jørgensen ${ }^{3}$ \\ Yasmine Amitay ${ }^{5}$ \\ Hilary Shmeeda ${ }^{5}$ \\ Alberto A Gabizon ${ }^{5}$ \\ Andreas $\mathrm{Kjær}^{3}$ \\ Thomas L Andresen ${ }^{1,2}$ \\ Anders E Hansen ${ }^{1-3}$ \\ 'Department of Micro- and \\ Nanotechnology, DTU Nanotech, \\ Technical University of Denmark, \\ DK-2800 Kongens Lyngby, Denmark; \\ ${ }^{2}$ Center for Nanomedicine and \\ Theranostics, Technical University of \\ Denmark, DK-2800 Kongens Lyngby, \\ Denmark; ${ }^{3}$ Cluster for Molecular \\ Imaging, Department of Biomedical \\ Sciences and Department of Clinical \\ Physiology, Nuclear Medicine \& \\ PET, University of Copenhagen and \\ Rigshospitalet, DK-2200 \& DK-2100, \\ Copenhagen, Denmark; ${ }^{4}$ Department \\ of Chemistry, DTU Chemistry, \\ Technical University of Denmark, \\ DK-2800 Kongens Lyngby, Denmark; \\ ${ }^{5}$ Shaare Zedek Medical Center and \\ Hebrew University - School of \\ Medicine, Jerusalem, Israel
}

Correspondence: Anders E Hansen Department of Micro- and Nanotechnology, DTU Nanotech, Technical University of Denmark, Anker Engelunds Vej I, DK-2800 Kongens Lyngby, Denmark

Tel +4560838079

Email aehan@nanotech.dtu.dk
Background: Active, ligand-mediated, targeting of functionalized liposomes to folate receptors (FRs) overexpressed on cancer cells could potentially improve drug delivery and specificity. Studies on folate-targeting liposomes (FTLs) have, however, yielded varying results and generally fail to display a clear benefit of FR targeting.

Method: Tumor accumulating potential of FTLs and NTLs were investigated in a FR overexpressing xenograft model by positron emission tomography/computed tomography imaging. Results: Tumors displayed significantly lower activity of FTLs than NTLs. Furthermore, FTLs displayed worse circulating properties and increased liver-accumulation than NTLs.

Conclusion: This study underlines that long-circulating properties of liposomes must be achieved to take advantage of EPR-dependent tumor accumulation which may be lost by functionalization. FR-functionalization negatively affected both tumor accumulation and circulation properties.

Keywords: liposomes, folate, cancer, imaging, PET, EPR

\section{Background}

Since the first description of the enhanced penetration and retention (EPR) effect in solid cancers, nano-sized drug delivery systems have been the subject for extensive research following their passive accumulation in malignant tissue. ${ }^{1}$ Liposome-based drug delivery systems is the most successful EPR-dependent nano-sized drug delivery systems due to highly flexible and versatile nature of liposomes. ${ }^{2}$ Despite their ability to achieve high level of fat accumulation in the cancerous tissue, liposomal drug delivery systems relying on passive uncontrolled drug release mechanisms have not revolutionized cancer therapy. Based on this, functionalized liposomes, which combine the passive EPR accumulation with selective uptake in cancer cells displaying a specific cell receptor, have been subject for intense research. Several studies and reviews conclude and state that actively targeting liposomes can significantly increase the amount of drug delivered to the target cell in comparison to the free-drug and passively targeting liposomal drug delivery systems. ${ }^{3}$ Even though these statements have been published extensively, very limited in vivo data support them. In vitro data provide extensive evidence of receptor targeting liposomes being taken up actively by cancer cells expressing the specific surface receptor. ${ }^{4-6}$ Notwithstanding these observations, we speculate that the tumor microenvironment creates an extensive barrier toward the trafficking and distribution of nano-sized molecules. This barrier may limit the access of active targeting liposomes to a very limited number of cancer cells in solid tumors and 
therefore reduce any therapeutic benefit relative to passively targeting liposome. The microregional intratumoral distribution of nano-sized liposomes has been illustrated to primarily be in the extracellular compartment adjacent to functional tumor blood vessels, which indicate that the proportion of cancer cells in direct contact with liposomes may be limited. ${ }^{7}$ This observation is further complicated by the existence of disorganized tumor vasculature and intervascular distance and highly therapy-resistant hypoxic tumor regions. ${ }^{8}$ Active targeting of liposome formulations may additionally make them more prone to recognition by the reticuloendothelial system (RES) thereby altering the circulating properties and reducing liposome availability for tumor accumulation. ${ }^{3,9}$ Despite the theoretical potential of active targeting liposomes the described obstacles may directly hinder the translation of in vitro efficacy to in vivo tumor models.

In the present study, we investigated the biodistribution and tumor-targeting potential of folate-targeting liposomes (FTLs). This was achieved using a highly flexible radiolabeled liposome platform, which was applied for somatostatin receptor targeting liposomes in human neuroendocrine cancer xenografts. ${ }^{10}$ Folate receptors (FRs) are highly expressed on a wide spectrum of cancers, including ovarian, lung, brain, head and neck, renal cell, and breast cancers and display relatively low expression on nontumorous tissues with the main exception being inflamed tissue. ${ }^{11,12}$ Importantly, FRs mediate endocytosis to nonlysosomal endosomal vesicles and therefore provides an attractive therapeutic target for intracellular liposomal drug delivery. ${ }^{13}$ The use of radiolabeled nontargeting liposomes (NTLs), radiolabeled FTLs, and functional positron emission tomography (PET) imaging combined with computed tomography (CT) allows for quantification of biodistribution and tumor accumulation levels of both formulations.

Previous studies evaluating the therapeutic potential of FTL drug delivery systems are limited and results are conflicting in regards to therapeutic advantage for intravenously administered formulations..$^{4,6,14-16}$ In comparison, intraperitoneal administration to ascites cancer models have improved the therapeutic efficacy of FTLs in comparison to NTLs. Thus, FTLs have potential, but this may require very directly accessible cancer cells. ${ }^{4,17}$ FTLs has been shown to be selectively taken up in the liver via receptor-mediated endocytosis in hepatic macrophages and display increased blood clearance relative to NTLs. ${ }^{9}$ Admistrating folic acid in mice intravenously or intraperitonealy has been shown to reduce hepatic uptake and increase circulating properties of liposomes, folate-targeting imaging agents, and folatetargeting liposomes. ${ }^{9,11}$
The current study was conducted in two stages; first, the biodistribution of copper-64 radiolabeled FTLs ( $\left.{ }^{64} \mathrm{Cu}-\mathrm{FTLs}\right)$ was compared to copper-64 radiolabeled NTLs $\left({ }^{64} \mathrm{Cu}-\mathrm{NTLs}\right)$ in mice bearing subcutaneous folate expressing KB-cell xenografts, and second, the effect of intravenous excess folic acid or vehicle on tumor uptake and biodistribution was compared for radiolabeled FTLs and NTLs.

\section{Methods}

\section{Preparation of ${ }^{64} \mathrm{Cu}$-radiolabeled FTLs and NTLs}

\section{Preparation of nontargeted liposomes}

NTLs entrapping the high-affinity copper chelator, DOTA, were prepared. Briefly, $50 \mathrm{mg} / \mathrm{mL}$ freeze-dried lipid powder (HSPC:Chol:DSPE-PEG2k 56.5:38.2:5.3, Lipoid) was dispersed in a buffer containing $10 \mathrm{mM}$ DOTA, $10 \mathrm{mM}$ HEPES, and $150 \mathrm{mM} \mathrm{NaCl}$ (pH 7.4). The lipid suspension was hydrated for 60 minutes at $65^{\circ} \mathrm{C}$ and subsequently sized to $100 \mathrm{~nm}$ using a Lipex thermo barrel extruder (Northern lipids). The nonencapsulated DOTA was removed by tangential flow filtration (Minimate ${ }^{\mathrm{TM}}$, Pall Corporation).

\section{Preparation of FTLs}

FTLs were prepared by postinsertion of $0.5 \mathrm{~mol} \%$ DSPEPEG5k-folate into NTLs containing DOTA. Briefly, $1.7 \mathrm{~mL}$ NTLs (9.4 mM lipid) was added to $0.5 \mathrm{mg}$ freeze-dried DSPEPEG5k-Folate powder, incubated for 20 minutes at $45^{\circ} \mathrm{C}$ with gentle shaking, then cooled on an ice bath, and checked for precipitates. The folate concentration was determined by UV-vis. Samples were diluted 1:9 in 10\% SDS solution and incubated for 30 minutes at $60^{\circ} \mathrm{C}$. Absorbance was measured at $282 \mathrm{~nm}$ and the folate concentration was determined via the molar extinction coefficient of DSPE-PEG5k-Folate $27,500 \mathrm{M}^{-1} \mathrm{~cm}^{-1}$ using the absorbance of NTLs as a reference. The liposome size and zeta potential were verified by DLS (ZetaPALS Brookhaven) and the lipid concentration was determined by ICP-MS (iCAP Q, Thermo Scientific). NTLs were produced having an average size of $110 \mathrm{~nm}$ (polydispersity index $=0.07$ ) and a zeta potential of $-5 \pm 1 \mathrm{mV}$. No change in size or zeta potential was determined for the FTLs upon post insertion of DSPE-PEG5k-Folate.

\section{Remote loading of ${ }^{64} \mathrm{CuCl}_{2}$ into NTLs and FTLs}

A total of $3.3 \mathrm{mM}$ liposomes were added to a vial containing dried ${ }^{64} \mathrm{CuCl}_{2}$. The liposome sample was incubated for 75 minutes at $65^{\circ} \mathrm{C}$ using constant stirring and then cooled to room temperature. The ${ }^{64} \mathrm{Cu}$ loading efficiency was determined by radio-HPLC and radio-thin-layer chromatography. ${ }^{18}$ 
The activity concentration was set to $60-75 \mathrm{MBq} / \mathrm{mL}$ corresponding to $12-15 \mathrm{MBq} /$ animal at the time of injection.

\section{Animal tumor model}

$\mathrm{KB}$ cells (human nasopharyngeal carcinoma cell line, CCL-17; known for overexpressing FRs ${ }^{19,20}$ were obtained from the American Type Culture Collection (ATCC, VA, USA). Cells were grown in monolayers in FFRPMI culture medium (modified RPMI without folic acid, vitamin $\mathrm{B}_{12}$, and phenol red) in a humidified atmosphere at $37^{\circ} \mathrm{C}$ containing $5 \% \mathrm{CO}_{2}$. The media was supplemented with $10 \%$ FBS, $2 \mathrm{mM}$ L-glutamine, $1 \mathrm{mM}$ sodium pyrovate, nonessential amino acids, $100 \mathrm{U} / \mathrm{mL}$ penicillin, and $100 \mu \mathrm{g} / \mathrm{mL}$ streptomycin.

Six-week-old female, athymic nude mice (NMRI nu/nu, Taconic Europe, Borup, Denmark) were allowed to acclimatize for 1 week before subcutaneous inoculation on both flanks with $\mathrm{KB}$ cancer cells $\left(1.5 \times 10^{6}\right.$ cells in $100 \mu \mathrm{L}$ serumfree media and Matrigel).

\section{Experimental setup}

Tumor xenografts were allowed to grow for 10 (study part 1) and 14 days (study part 2) prior to imaging. In study part 1 , animals were randomized into two groups to receive either; ${ }^{64} \mathrm{Cu}$-FTLs (n=7) or ${ }^{64} \mathrm{Cu}-\mathrm{NTLs}(\mathrm{n}=7)$. In study part 2 , animals were randomized into four groups: group $1,{ }^{64} \mathrm{Cu}$-FTLs preinjected with folic acid $(\mathrm{n}=8)$; group $2,{ }^{64} \mathrm{Cu}$-FTLs preinjected with vehicle ( $\mathrm{n}=8)$; group $3,{ }^{64} \mathrm{Cu}-\mathrm{NTLs}$ preloaded pre-injected with folic acid (n=5); and group $4,{ }^{64} \mathrm{Cu}-\mathrm{NTLs}$ preinjected with vehicle $(n=7)$. All experimental procedures were approved by and conducted under the guidelines of The Danish Animal Experiments Inspectorate.

\section{Imaging procedures}

All animals were anesthetized with a sevoflurane gas mixture and anesthetized mice had a tail vein catheter position to ensure strict intravenous injection of all formulations. All mice received $3.3 \mathrm{mM}$ of lipid. Two anesthetized mice were positioned side-by-side, separated by a $6 \mathrm{~mm}$ polystyrene block, on a heated platform for PET/CT imaging. PET/CT imaging was performed on a dedicated Inveon ${ }^{\circledR}$ small animal PET/CT system with CT-based PET image attenuation (Siemens Medical Systems, Malvern, PA, USA).

In study part $1,{ }^{64} \mathrm{Cu}$-labelled FTLs $\left({ }^{64} \mathrm{Cu}-\mathrm{FTLs}\right)$ and ${ }^{64} \mathrm{Cu}$-labelled NTLs $\left({ }^{64} \mathrm{Cu}\right.$-NTL) were administered as a bolus injection performed simultaneously with the start of a 10-minute dynamic PET acquisition. Three PET/CT sessions were conducted; a 10-minute dynamic acquisition commencing simultaneously with the bolus injection of liposomes, 5-minute static PET scan 3 hours after injection, and a 15-minute static PET scan 24 hours after injection. Following all PET scans, the imaging platform was moved automatically into the CT-scanner for anatomical imaging. The 10-minute dynamic PET scan was reconstructed into $10 \times 3$ seconds, $6 \times 5$ seconds, $4 \times 30$ seconds, and $7 \times 1$ minutes frames. PET scans were reconstructed using a maximum a posteriori (MAP) reconstruction algorithm (pixel size: $0.815 \times 0.815 \times 0.796 \mathrm{~mm}$ ) and attenuation correction was performed based on the corresponding CT scan.

For study part 2, mice were injected with $100 \mu \mathrm{g}$ of folic acid (Sigma-Aldrich, St Louis, MO, USA) suspended in $100 \mu \mathrm{L}$ of isotonic bicarbonate solution or $100 \mu \mathrm{L}$ of isotonic bicarbonate solution (vehicle) 2 minutes prior to infusing ${ }^{64} \mathrm{Cu}$-FTLs or ${ }^{64} \mathrm{Cu}-\mathrm{NTLs}$. Animals were allowed to cage rest until performing PET/CT scans. Scans were performed using the same PET/CT scanner and set up as study part 1.

Dynamic PET imaging was performed on two mice from groups 1, 2, and 4 using a MicroPET ${ }^{\circledR}$ Focus 120 (Siemens Medical Systems, Malvern, PA, USA). Mice were subsequently CT imaged using a MicroCAT-II system (Siemens Medical Systems, Malvern, PA, USA). The PET scan commenced simultaneously with the injection of ${ }^{64} \mathrm{Cu}$-labelled liposomes and dynamic images were collected for 2 hours. Only the static 24-hour PET/CT scans were performed for these mice. The 2-hour dynamic PET scans were reconstructed into $10 \times 3$ seconds, $6 \times 5$ seconds, $4 \times 30$ seconds, $7 \times 1$ minutes, and $22 \times 5$ minutes frames using a similar MAP reconstruction.

\section{Image analysis}

Image analysis was performed using commercially available Inveon software (Siemens Medical Systems, Malvern, PA, USA). Regions of interests (ROIs) were manually constructed based on the coregistered PET/CT images. The following ROIs were constructed: tumors (complete volume delineated), liver (multiple slices taking care to avoid the hilar region), spleen, muscle, and blood. Blood activity was estimated from a constructed volume of interest ROI covering the left ventricular lumen of the heart, except for the dynamically scanned mice from study part 2 , where an ROI was placed in the abdominal aorta. ROIs in the left ventricle and abdominal aorta were subsequently segmented to only include the voxels displaying above $80 \%$ of maximum activity with the original ROI. Time-activity curves (TACs) were constructed based on the dynamic images.

\section{Statistical analysis}

Statistical analysis was performed using Prism 5.0 software (GraphPad Software Inc., La Jolla, San Diego, CA, USA). 
Comparison between activity of ${ }^{64} \mathrm{Cu}-\mathrm{FTLs}$ and ${ }^{64} \mathrm{Cu}-$ NTLs in study part one was performed using unpaired $t$-test. For intergroup comparison between liposome activities in study part 2, one-way ANOVA with Tukey post hoc multiple comparison analysis was used. Data were tested to follow a normal distribution by Komolgorov-Smirnov test. Results reported mean \pm SEM (unless otherwise stated) and $P<0.05$ were considered statistically significant.

\section{Results}

\section{Tumor uptake of FR-targeted and nontargeted radiolabeled liposomes}

Tumor volume was compatible between groups displaying a mean size of $78.4 \pm 7.4 \mathrm{~mm}^{3}$. The PET ${ }^{64} \mathrm{Cu}$ activity levels were determined as \% injected dose per gram of tissue $(\% \mathrm{ID} / \mathrm{g})$ for the PET scans performed 3 hours and 24 hours postinjection (pi.). No tumor uptake was reported for the initial 10-minute dynamic PET scan. There was no statistical difference between mean and maximum activity between ${ }^{64} \mathrm{Cu}-F T L s(2.6 \% \pm 0.3 \% \mathrm{ID} / \mathrm{g}$ and $5.6 \% \pm 0.5 \% \mathrm{ID} / \mathrm{g})$ and ${ }^{64} \mathrm{Cu}-\mathrm{NTLs}(2.5 \% \pm 0.2 \% \mathrm{ID} / \mathrm{g}$ and $5.7 \% \pm 0.4 \% \mathrm{ID} / \mathrm{g})$ at 3 hours pi. (unpaired $t$-test) (Figure $1 \mathrm{~A}$ ). However, ${ }^{64} \mathrm{Cu}-$ FTLs displayed a statistically significant lower tumor-to-liver ratio compared with ${ }^{64} \mathrm{Cu}-\mathrm{NTLs}(P=0.0052)$. No difference between mean tumor-to-blood or tumor-to-spleen ratios was observed between groups (Figure 1B).

At the PET scan 24 hours pi., ${ }^{64} \mathrm{Cu}-\mathrm{NTLs}$ displayed a statistically significant higher mean $(7.8 \% \pm 0.5 \% \mathrm{ID} / \mathrm{g})$ and maximum $(18.2 \% \pm 1.5 \% \mathrm{ID} / \mathrm{g})$ tumor activity in

A

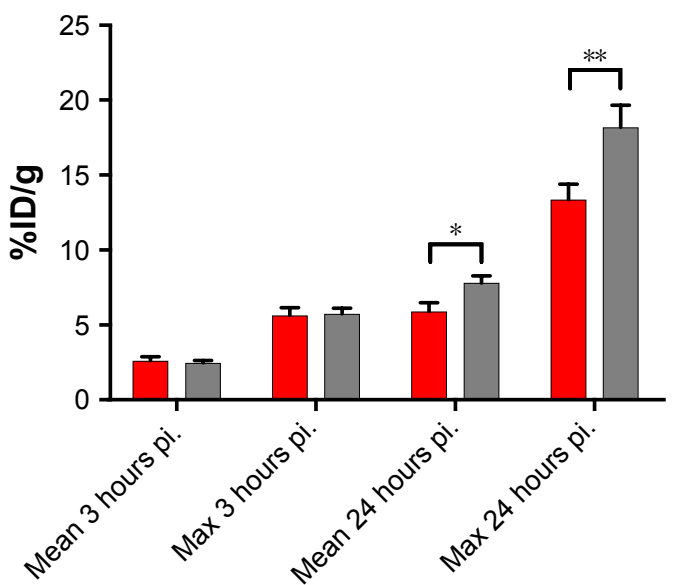

comparison to the mean $(5.9 \% \pm 0.6 \% \mathrm{ID} / \mathrm{g})$ and maximum $(13.4 \% \pm 1.0 \% \mathrm{ID} / \mathrm{g})$ tumor activity of ${ }^{64} \mathrm{Cu}-\mathrm{FTLs}(P=0.025$ and $P=0.011$ ) (Figure 1A). Mean tumor-to-blood ratio was statistically higher and tumor-to-liver ratio statistically lower for the ${ }^{64} \mathrm{Cu}$-NTLs compared to ${ }^{64} \mathrm{Cu}-\mathrm{FTLs}(P=0.005$ and $P=0.001$ ), whereas tumor-to-spleen ratio was compatible between groups (Figure 1B). This reflects the increased liver uptake and the decreased circulating half-life of ${ }^{64} \mathrm{Cu}$-FTLs.

\section{Biodistribution and uptake kinetics of FTLs and NTLs}

Mean liver activity of ${ }^{64} \mathrm{Cu}$-FTLs was statistically higher than ${ }^{64} \mathrm{Cu}-\mathrm{NTLs} 3$ hours pi. $(18.2 \% \pm 0.5 \% \mathrm{ID} / \mathrm{g}$ and $11.5 \% \pm 0.7 \% \mathrm{ID} / \mathrm{g}$, respectively, $P<0.0001)$ and at 24 hours pi. $(24.4 \% \pm 1.2 \% \mathrm{ID} / \mathrm{g}$ and $10.3 \pm 0.7$, respectively, $P<0.0001)$. No difference was observed for mean activity in the blood between ${ }^{64} \mathrm{Cu}-\mathrm{FTLs}$ and ${ }^{64} \mathrm{Cu}-\mathrm{NTLs} 3$ hours pi. $(28.7 \% \pm 1.3 \% \mathrm{ID} / \mathrm{g}$ and $29.6 \% \pm 1.7 \% \mathrm{ID} / \mathrm{g}$, respectively). However, 24 hours pi. activity of ${ }^{64} \mathrm{Cu}$-FTLs was significantly lower than ${ }^{64} \mathrm{Cu}-\mathrm{NTLs}$ $(4.9 \% \pm 0.3 \% \mathrm{ID} / \mathrm{g}$ and $9.1 \% \pm 1.0 \% \mathrm{ID} / \mathrm{g}, P=0.001)$. On the contrary, splenic uptake of ${ }^{64} \mathrm{Cu}-\mathrm{FTLs}$ was significantly lower 24 hours pi. relative to ${ }^{64} \mathrm{Cu}-\mathrm{NTLs}(8.5 \% \pm 0.6 \% \mathrm{ID} / \mathrm{g}$ and $12.3 \% \pm 1.5 \% \mathrm{ID} / \mathrm{g}$, respectively, $P=0.032$ ), whereas no significant difference was observed for mean activity 3 hours pi. $(10.4 \% \pm 0.4 \% \mathrm{ID} / \mathrm{g}$ and $13.2 \% \pm 1.5 \% \mathrm{ID} / \mathrm{g}$, respectively). However, the potential influence of blood volume in the spleen and the difference observed in blood activity 24 hours pi., must be taken into consideration and differences

\section{B}

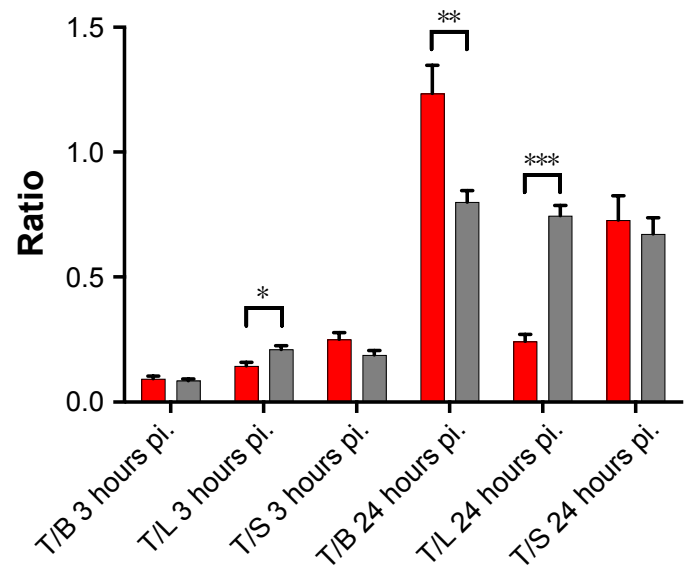

\section{$\square$ FTLs $\square$ NTLs}

Figure I Accumulation of FTL and NTL in tumors.

Notes: (A) PET tumor activity of ${ }^{64} \mathrm{Cu}-\mathrm{FTLs}$ and ${ }^{64} \mathrm{Cu}-\mathrm{NTLs} 3$ hours pi and 24 hours pi in tumors. Asterisks indicate statistically different means between mean and maximum activity of ${ }^{64} \mathrm{Cu}-\mathrm{FTLs}$ and ${ }^{64} \mathrm{Cu}-\mathrm{NTLs}\left({ }^{* P}=0.025\right.$, ${ }^{* * P}=0.0 \mathrm{I}$ I, unpaired Student's $t$-test). (B) Tumor (T) to blood (B), liver (L), and spleen (S) activity ratios of ${ }^{64} \mathrm{Cu}-\mathrm{FTLs}$ and ${ }^{64} \mathrm{Cu}-\mathrm{NTLs} 3$ hours pi. and 24 hours pi. ( $* P=0.0052$, $* * P=0.005$, $* * * P=0.00 \mathrm{I}$, unpaired Student's $t$-test, bars represent mean \%ID/g $\left.\pm S E M\right)$.

Abbreviations: NTL, nontargeting liposomes; FTL, folate-targeting liposomes; SEM, standard error of mean; pi., postinjection. 
A

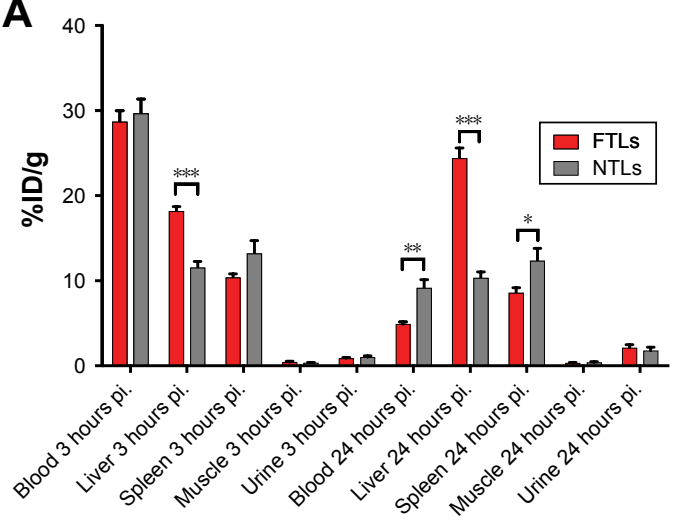

B

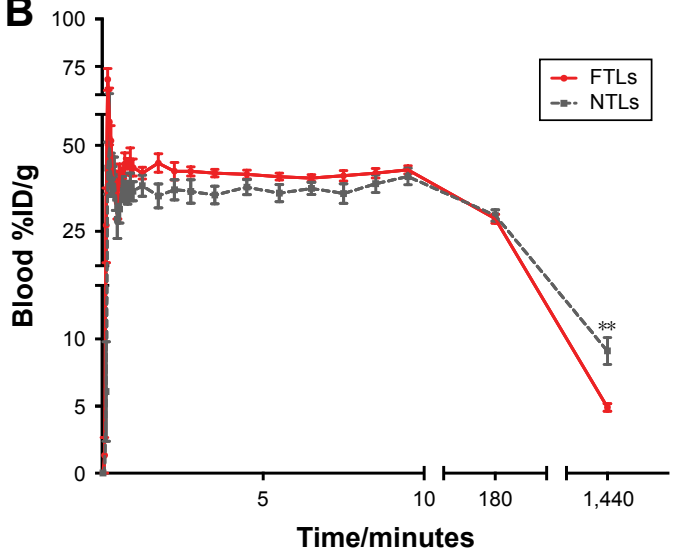

C

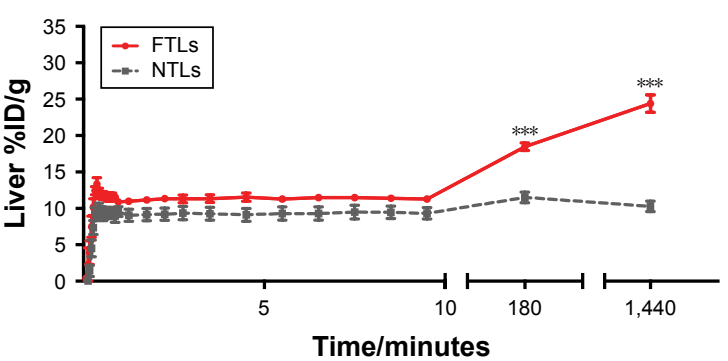

D

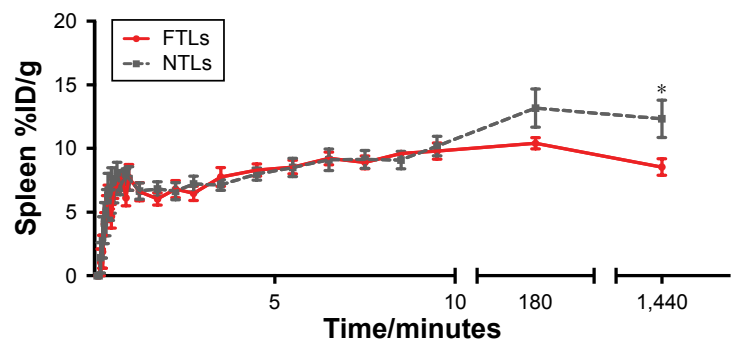

E

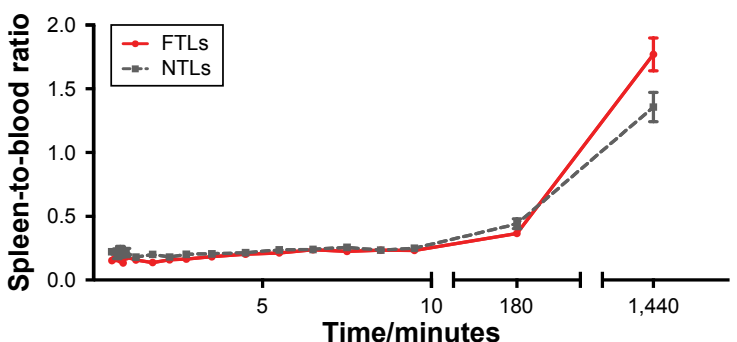

Figure 2 Biodistribution of FTLs and NTLs.

Notes: (A) Biodistribution of ${ }^{64} \mathrm{Cu}-\mathrm{FTLs}$ and ${ }^{64} \mathrm{Cu}-\mathrm{NTLs} 3$ hours pi. and 24 hours pi. Asterisks indicate statistically different means between the mean and maximum activity of ${ }^{64} \mathrm{Cu}-\mathrm{FTLs}$ and ${ }^{64} \mathrm{Cu}-\mathrm{NTL}$ (bars represent mean \%ID/g士SEM). (B-D) TAC of blood (B), liver (C), and spleen. (D) activity of ${ }^{64} \mathrm{Cu}-\mathrm{FTLs}$ and ${ }^{64} \mathrm{Cu}-\mathrm{NTL}$ during a $10-\mathrm{minute}$ dynamic PET scan immediately after injection and static 3 hours and 24 hours pi. scans (mean \pm SEM). (E) TAC of spleen-to-blood ratios for ${ }^{64} \mathrm{Cu}-\mathrm{FTLs}$ and ${ }^{64} \mathrm{Cu}-\mathrm{NTLs}$ during the initial I0-minute dynamic PET scan and static 3 hours and 24 hours pi. scans (mean \pm SEM, $* P=0.032, * * P=0.001$, ***P $<0.0000$ I, unpaired Student's $t$-test).

Abbreviations: NTL, nontargeting liposomes; FTL, folate-targeting liposomes; TAC, time-activity curves; PET, positron emission tomography; SEM, standard error of mean.

can probably not solely be attributed to splenic uptake. No difference was observed for uptake levels in muscle 3 in 24 hours pi. (Figures $2 \mathrm{~A}$ and 3).

Dynamic uptake of the liposomal formulation was determined from the initial dynamic PET scan and scans 3 and 24 hours pi. (Figure 2B). TACs illustrate the increased blood clearance and hepatic accumulation of ${ }^{64} \mathrm{Cu}$-FTLs relative to ${ }^{64} \mathrm{Cu}-\mathrm{NTLs}$ (Figure 2B and C). Interestingly, splenic TAC illustrates the higher activity of ${ }^{64} \mathrm{Cu}$-NTLs rather ${ }^{64} \mathrm{Cu}$-FTLs 24 hours pi. (Figure 2D). However, constructing a TAC of the spleen-to-blood ratio reverses this observation, which could indicate that the observed difference could be attributed to the higher activity of ${ }^{64} \mathrm{Cu}-\mathrm{NTL}$ in the blood (Figure 2E).

\section{Tumor uptake of FTLs after folic acid coadministration}

Tumor volume was compatible between the four groups of mice in the study part $2\left(119.6 \pm 12.9 \mathrm{~mm}^{3}\right)$, although slightly larger than the mean tumor volume of the tumors in study part 1 following a longer growth period. No difference in mean tumor activity between the group receiving excess folic acid or vehicle and ${ }^{64} \mathrm{Cu}$-FTLs or ${ }^{64} \mathrm{Cu}$-NTLs was observed 3 hours pi. (Figure 4A). However, 24 hours pi., tumors in both ${ }^{64} \mathrm{Cu}$-NTLs groups display a higher mean and maximum activity of radiolabeled liposomes relative to groups receiving ${ }^{64} \mathrm{Cu}$-FTLs $(P<0.0001)$. Treatment with folic acid or vehicle mediated no change in tumor uptake between the ${ }^{64} \mathrm{Cu}$-FTLs groups or between the folic acid and vehicle ${ }^{64} \mathrm{Cu}-\mathrm{NTL}$ mice (Figure 4A).

\section{Effect of coadministration of excess folic acid on biodistribution and uptake kinetics of FTLs and NTLs}

The coadministration of excess folic acid reduced the mean liver activity of ${ }^{64} \mathrm{Cu}-\mathrm{FTLs} 3$ hours pi. to $12.85 \% \pm 0.9 \% \mathrm{ID} / \mathrm{g}$, which was not statistically higher than the folic acid $(11.7 \% \pm 0.9 \% \mathrm{ID} / \mathrm{g})$ and vehicle $(11.3 \% \pm 0.4 \% \mathrm{ID} / \mathrm{g})$ treated ${ }^{64} \mathrm{Cu}$-NTLs mice. Liver activity in ${ }^{64} \mathrm{Cu}$-FTLs mice receiving vehicle was significantly higher $(18.3 \% \pm 1.6 \% \mathrm{ID} / \mathrm{g})$ compared to all other groups $(P=0.0005)$. However, the 


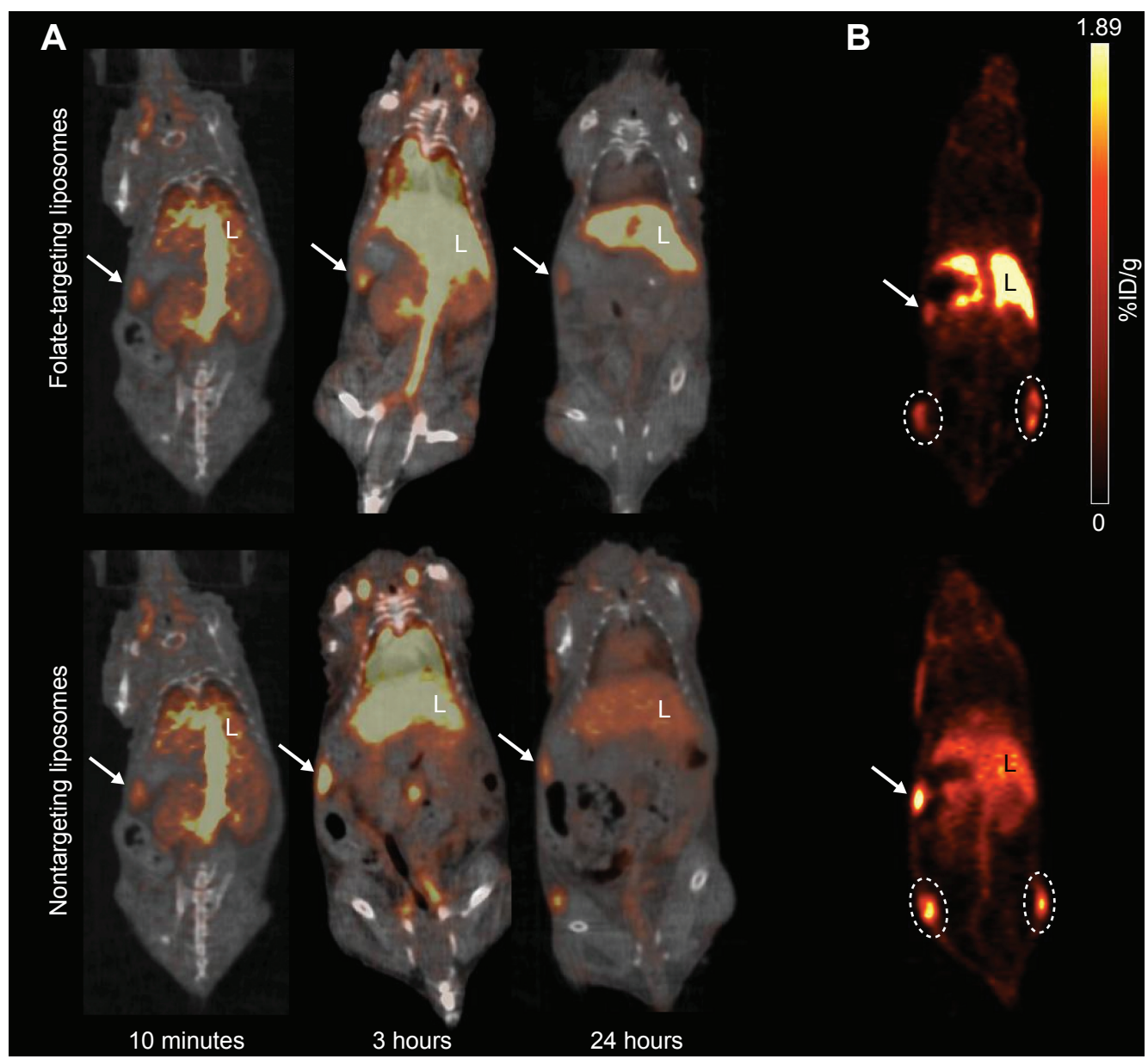

Figure 3 PET/CT of FTLs and NTLs in tumor-bearing mice.

Notes: (A) ${ }^{64} \mathrm{Cu}$-liposome PET/CT images of ${ }^{64} \mathrm{Cu}$-FTLs 10 minutes, 3 hours, and 24 hours pi. (top images) and ${ }^{64} \mathrm{Cu}-\mathrm{NTLs} 10$ minutes, 3 hours, and 24 hours pi. (bottom images). Liver (L), spleen (arrow). (B) ${ }^{64} \mathrm{Cu}$-liposome PET images 24 hours pi. of tumor uptake of ${ }^{64} \mathrm{Cu}-\mathrm{FTLs}$ (top image) and ${ }^{64} \mathrm{Cu}-\mathrm{NTLs}$ (bottom image). Left and right flank tumors are encircled in white, liver (L), spleen (arrow).

Abbreviations: FTL, folate-targeting liposomes; NTL, nontargeting liposomes; PET/CT, positron emission tomography/computed tomography.

effect of excess folic acid on liver uptake was no longer evident 24 hours pi., where mean liver activity of ${ }^{64} \mathrm{Cu}-$ FTLs in the folic acid $(19.3 \% \pm 0.9 \% \mathrm{ID} / \mathrm{g})$ and vehicle group $(22.4 \% \pm 1.1 \% \mathrm{ID} / \mathrm{g})$ was no longer significantly different and both were significantly higher than ${ }^{64} \mathrm{Cu}-\mathrm{NTLs}$ in the folic acid $(11.6 \% \pm 0.6 \% \mathrm{ID} / \mathrm{g})$ and vehicle group $(11.2 \% \pm 0.8 \% \mathrm{ID} / \mathrm{g})(P<0.0001)$ (Figure 4B). Additionally, liver activities during the first 120 minutes pi. of FTLs coadministered with folic acid resembled NTLs administered with a vehicle while FTLs with vehicle displayed higher liver activity (Figure 4C).

Blood activity of radiolabeled liposomes was not statistically different between groups 3 hours pi. The blood activity levels 24 hours pi. were statistically lower in both ${ }^{64} \mathrm{Cu}$-FTLs groups (folic acid; $2.8 \% \pm 0.3 \% \mathrm{ID} / \mathrm{g}$ and vehicle; $2.8 \% \pm 0.3 \% \mathrm{ID} / \mathrm{g}$ ) in comparison to ${ }^{64} \mathrm{Cu}-\mathrm{NTLs}$ (folic acid; $6.1 \% \pm 0.4 \% \mathrm{ID} / \mathrm{g}$ and vehicle; $6.3 \% \pm 0.2 \% \mathrm{ID} / \mathrm{g})(P<0.0001)$. The activity of radiolabeled liposomes in the spleen was not statistically different between any of the groups 3 hours pi. nor 24 hours pi. (Figure 4B).

Dynamic PET scanning was performed to evaluate the influence of coadministration of excess folic acid on the initial liver uptake of mice receiving ${ }^{64} \mathrm{Cu}$-FTLs and excess folic acid or vehicle and ${ }^{64} \mathrm{Cu}-\mathrm{NTL}$ plus vehicle. Comparison of the liver TAC indicates that coadministration of excess folic acid delays the initial liver activity of ${ }^{64} \mathrm{Cu}$ FTLs to a level comparable to ${ }^{64} \mathrm{Cu}-\mathrm{NTLs}$. However, after the first-hour uptake rate increases to a level comparable to that of ${ }^{64} \mathrm{Cu}$-FTLs vehicle-treated mice. TACs are based on only two mice in each group and they must be carefully interpreted.

\section{Discussion}

Despite the generally positive effects of FTLs in vitro, studies conducted in vivo have failed to yield compatible improved efficacy. This limited success of intravenously administered 

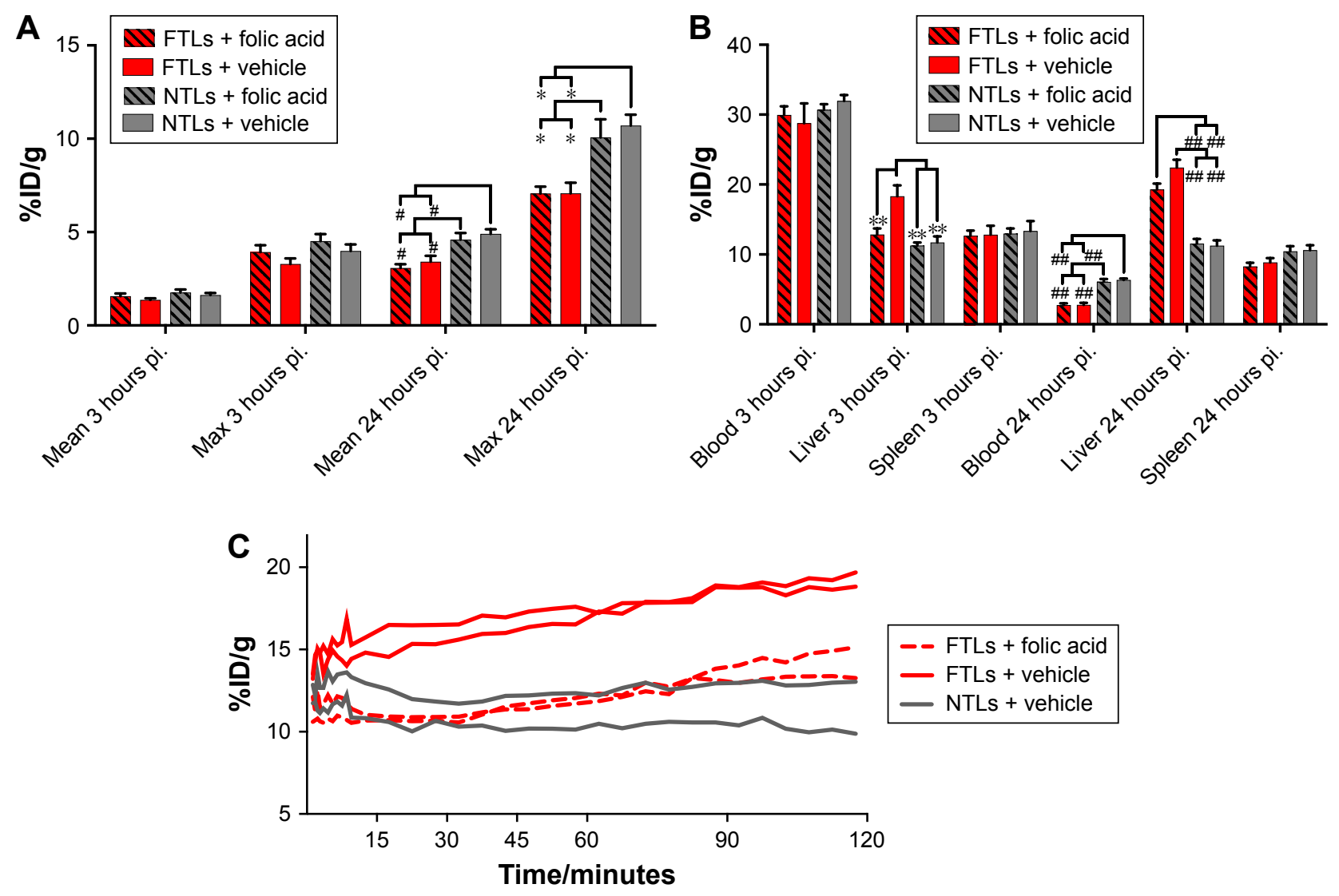

Figure 4 Biodistribution of FTLs and NTLs in competition with folic acid.

Notes: (A) PET tumor activity of ${ }^{64} \mathrm{Cu}-\mathrm{FTLs}$ and ${ }^{64} \mathrm{Cu}-\mathrm{NTLs}$ with folic acid or vehicle coadministration 3 hours pi. and 24 hours pi. Tumor mean and maximum activity was statistically higher for ${ }^{64} \mathrm{Cu}-\mathrm{NTL}$ in comparison to ${ }^{64} \mathrm{Cu}-\mathrm{FTL}$ independent of administration of folic acid $\left({ }^{\#} \mathrm{P}=0.0005\right.$ and $\left.* \mathrm{P}<0.000 \mathrm{I}\right)$. (B) Biodistribution of ${ }^{64} \mathrm{Cu}-\mathrm{FTLs}$ and ${ }^{64} \mathrm{Cu}-\mathrm{NTLs}$ with folic acid or vehicle coadministration 3 hours pi. and 24 hours pi. Folic acid predosing was able to reduce early (3 hours pi.) mean liver activity of ${ }^{64} \mathrm{Cu}-\mathrm{FTL}$ to the level of ${ }^{64} \mathrm{Cu}-\mathrm{NTL}$ liver activity. The liver activity of vehicle administered ${ }^{64} \mathrm{Cu}-\mathrm{FTL}$ was significantly higher than ${ }^{64} \mathrm{Cu}-\mathrm{NTL}$ (vehicle and folic acid predosed) and folic acid predosed ${ }^{64} \mathrm{Cu}-\mathrm{FTL}\left({ }^{*} * \mathrm{P}<0.0 \mathrm{I}\right)$. Independent of folic acid or vehicle predosing the blood mean activity 24 hours pi. was significantly higher for ${ }^{64} \mathrm{Cu}-\mathrm{NTL}$ in comparison to the

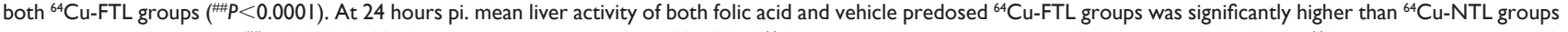
independent of predosing ( ${ }^{\# P<0.000 I) . ~(C) ~ 2-h o u r ~ d y n a m i c ~ l i v e r ~ a c t i v i t y ~(\% I D / g) ~ o f ~}{ }^{64} \mathrm{Cu}-\mathrm{FTL}$ and folic acid predosing $(n=2)$ or vehicle $(n=2)$ and ${ }^{64} \mathrm{Cu}-\mathrm{NTL}$ and vehicle ( $\left.\mathrm{n}=2\right)$ on PET scans commencing simultaneously with the injection of radiolabeled liposomes (bars represent mean \%ID/g \pm SEM. All comparisons were performed using one-way ANOVA analysis and post hoc Tukey multiple comparison analysis).

Abbreviations: FTL, folate-targeting liposomes; NTL, nontargeting liposomes; PET, positron emission tomography; SEM, standard error of mean; pi., postinjection.

liposome formulations targeting FRs was the basis for the present study, which aimed to provide direct quantitative information on tumor accumulation and circulating characteristics of FTLs by high sensitive and quantitative PET imaging. ${ }^{4,6,21}$

The presented data illustrate the impact of the reduced circulating half-life of FTLs on tumor accumulation and that actively targeting FRs, highly expressed on cancer cells, does not improve overall delivery. FTLs were rapidly taken up in the liver which was already observable 3 hours pi. The high level of liver uptake has previously been reported for both FTLs and folate-targeting imaging agents., ${ }^{9}, 11$

This study was conducted on relatively small solid tumors. This was based on the previously reported improved therapeutic efficacy of encapsulated doxorubicin in FTLs in comparison to NTLs in small KB xenografts inoculated in the footpad of mice, ${ }^{4}$ which suggested a potential targeting possibility in small tumors. The larger tumors in the second part of the study displayed slightly lower mean tumor uptake, supporting previous reports of lower EPR-based liposome uptake with increasing tumor size. ${ }^{22}$

Although a folate-restricted diet might be expected to boost FR expression, it has been shown not to influence folate binding of KB xenografts and M109 tumors in mice $^{9}$, and thus this study was conducted without feeding mice folate-restricted diets. Additionally, low-folate diets were shown to increase tissue retention of folate-targeted radiopharmaceuticals ${ }^{23,24}$ which would further deplete circulating FTLs.

The effect of blocking uptake by liver by coadministering excess folic acid to improve the circulating properties of FTLs was investigated. This approach has been identified to decrease uptake in liver and other tissues expressing high levels of FRs ${ }^{11}$ and a similar folate preinjection approach has 
been safely performed in human ovarian and endometrial cancer patients. ${ }^{25}$ Thus, intravenous preinjection of excess folate had acceptable translational potential and was chosen over the intraperitoneal administration of very high doses of folic acid, which may result in a more prolonged blockade of hepatic receptors. ${ }^{24} \mathrm{~A}$ preinjection of excess folic acid has, additionally, been shown to decrease tumor accumulation of folate-targeting radiotracers ${ }^{11,26}$ and may have no effect on liposome accumulation in tumor. ${ }^{9}$ In this study, the results of folic acid coadministration demonstrate no difference between FTLs and NTLs uptake levels in tumors of mice pretreated with folic acid or vehicle. Liver uptake was only significantly reduced in folic acid injected mice in the 3 hours PET scan, indicating that the blockade is short lived and insufficient for liposomes with long-circulating half-lives. To increase the circulating half-life of FTLs, multiple doses or long-acting folic acid may be needed which, following the reported blockade of tumor cell receptors by the excess folic acid administration, could directly inhibit the cancer cell-targeting strategy. Importantly, the influence of folate-mediated liver uptake in humans injected with FTLs remains to be determined. However, the liver is the primary storage organ for folate and FRs are expressed by activated human macrophages. Macrophage-mediated uptake and elimination may therefore also be an important issue for circulatory properties in humans. ${ }^{27-29}$ Alternatively, administration of antifolates (eg, pemetrexed) has been performed in conjunction with FR-targeting agents and shown to decrease renal uptake without affecting tumor uptake. ${ }^{30}$ Administration of pemetrexed may, however, be associated with unwanted side effects, especially in patients already undergoing chemotherapy.

The results of this study indicate that the EPR effect and circulating properties of liposome formulations determine the liposome levels that may be achieved, independently of active tumor cell receptor targeting. This statement is supported by the previously observed reduced, but more compatible, uptake levels of somatostatin receptor targeting liposomes relative to NTLs. ${ }^{10}$

The results demonstrate that the active targeting of FRs on human cancer cells with known high expression decreases the overall liposome uptake within solid tumor xenografts. Thus, drug delivery by liposomes targeting FRs significantly decrease the total amount of liposomes delivered to solid tumors. It is therefore of key importance to include a nontargeting liposomal comparator when evaluating anticancer therapy based on targeting liposomes if statements on the improved efficiency of active targeting are to be valid. Despite the lack of valid comparators, FTLs have been purported to provide improved therapeutic efficacy based on their targeting properties although this effect is primarily attributable to an EPR-based improved drug delivery within tumors. ${ }^{31}$

The presented data do not provide information on the uptake levels achieved in single cancer cells within the solid tumor mass between targeting and NTLs. Several studies and reviews highlight the increased cellular uptake by active targeting as a method to increase the amount of drug delivered and thus improve the therapeutic efficacy. ${ }^{3}$

This consideration might be attractive if all cells within solid tumors were readily accessible for nano-sized particles. However this does not seem to be the case. ${ }^{7}$ Active targeting to the more readily available neo-angiogenic tumor vasculature is far more appealing. Several attractive vascular targets are probably directly accessible to circulating liposomes and could allow accumulation and subsequent triggered drug release within the vasculature to increase loco-regional drug concentration. ${ }^{32,33}$ FTLs may have therapeutic potential beyond the direct targeting of FR expressing cancer cells as important associations between tumor-associated macrophages (TAMs) expressing FR- $\beta$ and malignant characteristics has been identified. TAMs play prominent roles in angiogenesis, cancer cell invasion, and extravasation. FR- $\beta$ expressing TAM has additionally been shown to mediate potent immunosuppressive functions. ${ }^{34-36}$ Importantly, with respect to the perivascular accumulation liposomes, the TAMs reside in the perivascular compartment thus making them potential targets that could be reached by FTLs. ${ }^{34,37}$ The majority FR- $\beta$ expressing TAM in human pancreatic cancer has additionally been shown to express vascular endothelial growth factor which increases vascular permeability and therefore potentially improved liposome extravasation to perivascular areas harboring FR- $\beta$ TAMs. ${ }^{34,38}$

Notwithstanding the challenges faced EPR-based liposome accumulation in solid cancer remains an attractive methodology for drug delivery, however, achieving improved targeting and triggered release within the accessible extracellular compartments remain an important goal.

Directly targeting specific cancer cell receptors, including FRs, has proven successful in numerous in vitro studies. ${ }^{39-42}$ Varying therapeutic effects of FR-targeting in tumor models has been observed in in vivo studies, ranging from promising to discouraging results ${ }^{4,6,14,15,43,44}$ However, other in vivo studies has also, in agreement with the current study, targeted FR with liposomes with poor tumor accumulation compared to controls..$^{9,12,45}$ Although results by previous studies are 
somewhat contradicting, it may be partly explained by differences in tumor models, formulations, and other experimental details and, as previously stated, be partly explained by differences in cellular uptake. Previous in vivo studies have mainly used ${ }^{3} \mathrm{H}$ - or fluorophore-imaging to assess biodistribution while the current study included PET/CT imaging to obtain more dynamic and sensitive results. Furthermore, this study demonstrates the importance of in vivo studies to demonstrate the actual targeting potential.

In conclusion, targeting FRs provided no improvement of the overall liposome delivery to solid KB xenografts determined by PET. Although only FTLs were included in the present study. It clearly demonstrates that surface modifications to liposomes must be carefully evaluated as this may significantly influence the circulation and biodistribution. Any observed improved in vitro cytotoxic efficacy must, therefore, be carefully evaluated as this improved effect, in comparison to long-circulating controls, can easily be lost following significant difference in EPR-dependent tumor accumulation. Further studies are warranted to fully elucidate the potential of various ligands targeting cancer cell receptors and subsequent intratumoral microregional distribution. Importantly, the present study was conducted in a murine xenograft model, which may display a far less complicated tumor environment than clinical cancers and therefore potentially illustrate the problems targeted drug delivery systems faced in clinical cancers. The targeting and therapeutic potential of FTLs may benefit from investigations in additional animal models with a folate metabolism that better simulate human folate metabolism to elucidate their and therapeutic potential fully.

\section{Disclosure}

The authors report no conflicts of interest in this work.

\section{References}

1. Matsumura Y, Maeda H. A new concept for macromolecular therapeutics in cancer chemotherapy: mechanism of tumoritropic accumulation of proteins and the antitumor agent smancs. Cancer Res. 1986;46(12 Pt 1): 6387-6392.

2. Petersen AL, Hansen AE, Gabizon A, Andresen TL. Liposome imaging agents in personalized medicine. Adv Drug Deliv Rev. 2012;64(13): 1417-1435.

3. Noble GT, Stefanick JF, Ashley JD, Kiziltepe T, Bilgicer B. Ligandtargeted liposome design: challenges and fundamental considerations. Trends Biotechnol. 2014;32(1):32-45.

4. Gabizon A, Tzemach D, Gorin J, et al. Improved therapeutic activity of folate-targeted liposomal doxorubicin in folate receptor-expressing tumor models. Cancer Chemother Pharmacol. 2010;66(1):43-52.

5. Saul JM, Annapragada A, Natarajan JV, Bellamkonda RV. Controlled targeting of liposomal doxorubicin via the folate receptor in vitro. J Control Release. 2003;92(1-2):49-67.
6. Riviere K, Huang Z, Jerger K, Macaraeg N, Szoka FC. Antitumor effect of folate-targeted liposomal doxorubicin in KB tumor-bearing mice after intravenous administration. J Drug Target. 2011;19(1):14-24.

7. Yuan F, Leunig M, Huang SK, Berk DA, Papahadjopoulos D, Jain RK. Microvascular permeability and interstitial penetration of sterically stabilized (stealth) liposomes in a human tumor xenograft. Cancer Res. 1994;54(13):3352-3356.

8. Vaupel P, Mayer A. Hypoxia in cancer: significance and impact on clinical outcome. Cancer Metastasis Rev. 2007;26(2):225-239.

9. Gabizon A, Horowitz AT, Goren D, Tzemach D, Shmeeda H, Zalipsky S. In vivo fate of folate-targeted polyethylene-glycol liposomes in tumorbearing mice. Clin Cancer Res. 2003;9(17):6551-6559.

10. Petersen AL, Binderup T, Jølck RI, et al. Positron emission tomography evaluation of somatostatin receptor targeted $64 \mathrm{Cu}$-TATE-liposomes in a human neuroendocrine carcinoma mouse model. J Control Release. 2012;160(2):254-263.

11. Müller C, Forrer F, Schibli R, Krenning EP, de Jong M. SPECT study of folate receptor-positive malignant and normal tissues in mice using a novel 99mTc-radiofolate. J Nucl Med. 2008;49(2):310-317.

12. Poh S, Chelvam V, Low PS. Comparison of nanoparticle penetration into solid tumors and sites of inflammation: studies using targeted and nontargeted liposomes. Nanomedicine. 2015;10(9):1439-1449.

13. Yang J, Chen H, Vlahov IR, Cheng JX, Low PS. Evaluation of disulfide reduction during receptor-mediated endocytosis by using FRET imaging. Proc Natl Acad Sci U S A. 2006;103(37):13872-13877.

14. Pan XQ, Zheng X, Shi G, Wang H, Ratnam M, Lee RJ. Strategy for the treatment of acute myelogenous leukemia based on folate receptor beta-targeted liposomal doxorubicin combined with receptor induction using all-trans retinoic acid. Blood. 2002;100(2):594-602.

15. Pan XQ, Wang H, Lee RJ. Antitumor activity of folate receptor-targeted liposomal doxorubicin in a KB oral carcinoma murine xenograft model. Pharm Res. 2003;20(3):417-422.

16. Yamada A, Taniguchi Y, Kawano K, Honda T, Hattori Y, Maitani Y. Design of folate-linked liposomal doxorubicin to its antitumor effect in mice. Clin Cancer Res. 2008;14(24):8161-8168.

17. Pan XQ, Lee RJ. In vivo antitumor activity of folate receptor-targeted liposomal daunorubicin in a murine leukemia model. Anticancer Res. 2005;25(1A):343-346.

18. Henriksen JR, Petersen AL, Hansen AE, et al. Remote Loading of (64) $\mathrm{Cu}(2+)$ into liposomes without the use of ion transport enhancers. ACS Appl Mater Interfaces. 2015;7(41):22796-22806.

19. Forster MD, Ormerod MG, Agarwal R, Kaye SB, Jackman AL. Flow cytometric method for determining folate receptor expression on ovarian carcinoma cells. Cytometry A. 2007;71(11):945-950.

20. Siwowska K, Schmid RM, Cohrs S, Schibli R, Müller C. Folate receptorpositive gynecological cancer cells: in vitro and in vivo characterization. Pharmaceuticals. 2017;10(3):E72.

21. Tong L, Chen W, Wu J, Li H. Folic acid-coupled nano-paclitaxel liposome reverses drug resistance in SKOV3/TAX ovarian cancer cells. Anticancer Drugs. 2014;25(3):244-254.

22. Harrington KJ, Mohammadtaghi S, Uster PS, et al. Effective targeting of solid tumors in patients with locally advanced cancers by radiolabeled pegylated liposomes. Clin Cancer Res. 2001;7(2):243-254.

23. Leamon CP, Reddy JA, Dorton R, et al. Impact of high and low folate diets on tissue folate receptor levels and antitumor responses toward folatedrug conjugates. J Pharmacol Exp Ther. 2008;327(3):918-925.

24. Gabizon A, Shmeeda H, Horowitz AT, Zalipsky S. Tumor cell targeting of liposome-entrapped drugs with phospholipid-anchored folic acidPEG conjugates. Adv Drug Deliv Rev. 2004;56(8):1177-1192.

25. Siegel BA, Dehdashti F, Mutch DG, et al. Evaluation of 111 In-DTPAfolate as a receptor-targeted diagnostic agent for ovarian cancer: initial clinical results. J Nucl Med. 2003;44(5):700-707.

26. Ross TL, Honer M, Müller C, Groehn V, Schibli R, Ametamey SM. A new 18F-labeled folic acid derivative with improved properties for the PET imaging of folate receptor-positive tumors. J Nucl Med. 2010; 51(11):1756-1762. 
27. Hoppner K, Lampi B. Folate levels in human liver from autopsies in Canada. Am J Clin Nutr. 1980;33(4):862-864.

28. Müller A, Beck K, Rancic Z, et al. Imaging atherosclerotic plaque inflammation via folate receptor targeting using a novel $18 \mathrm{~F}$-folate radiotracer. Mol Imaging. 2014;13(2):1-11.

29. van der Heijden JW, Oerlemans R, Dijkmans BA, et al. Folate receptor beta as a potential delivery route for novel folate antagonists to macrophages in the synovial tissue of rheumatoid arthritis patients. Arthritis Rheum. 2009;60(1):12-21.

30. Müller C, Schibli R. Prospects in folate receptor-targeted radionuclide therapy. Front Oncol. 2013;3:249.

31. Morton SW, Lee MJ, Deng ZJ, et al. A nanoparticle-based combination chemotherapy delivery system for enhanced tumor killing by dynamic rewiring of signaling pathways. Sci Signal. 2014;7(325):ra44.

32. Petersen AL, Binderup T, Rasmussen P, et al. 64Cu loaded liposomes as positron emission tomography imaging agents. Biomaterials. 2011; 32(9):2334-2341.

33. Chen Q, Tong S, Dewhirst MW, Yuan F. Targeting tumor microvessels using doxorubicin encapsulated in a novel thermosensitive liposome. Mol Cancer Ther. 2004;3(10):1311-1317.

34. Kurahara H, Takao S, Kuwahata T, et al. Clinical significance of folate receptor $\beta$-expressing tumor-associated macrophages in pancreatic cancer. Ann Surg Oncol. 2012;19(7):2264-2271.

35. Puig-Kröger A, Sierra-Filardi E, Domínguez-Soto A, et al. Folate receptor beta is expressed by tumor-associated macrophages and constitutes a marker for M2 anti-inflammatory/regulatory macrophages. Cancer Res. 2009;69(24):9395-9403.

36. Quail DF, Joyce JA. Microenvironmental regulation of tumor progression and metastasis. Nat Med. 2013;19(11):1423-1437.
37. Wyckoff JB, Wang Y, Lin EY, et al. Direct visualization of macrophageassisted tumor cell intravasation in mammary tumors. Cancer Res. 2007; 67(6):2649-2656.

38. Chen XL, Nam JO, Jean C, et al. VEGF-induced vascular permeability is mediated by FAK. Dev Cell. 2012;22(1):146-157.

39. Patil Y, Shmeeda H, Amitay Y, Ohana P, Kumar S, Gabizon A. Targeting of folate-conjugated liposomes with co-entrapped drugs to prostate cancer cells via prostate-specific membrane antigen (PSMA). Nanomedicine. 2018;14(4):1407-1416.

40. Watanabe K, Kaneko M, Maitani Y. Functional coating of liposomes using a folate-polymer conjugate to target folate receptors. Int $J$ Nanomedicine. 2012;7:3679-3688.

41. Guo B, Xu D, Liu X, Yi J. Enzymatic synthesis and in vitro evaluation of folate-functionalized liposomes. Drug Des Devel Ther. 2017; 11:1839-1847.

42. Wang F, Chen Y, Zhang D, et al. Folate-mediated targeted and intracellular delivery of paclitaxel using a novel deoxycholic acid-Ocarboxymethylated chitosan-folic acid micelles. Int J Nanomedicine. 2012;7:325-337.

43. Ke X, Lin W, Li X, Wang H, Xiao X, Guo Z. Synergistic dual-modified liposome improves targeting and therapeutic efficacy of bone metastasis from breast cancer. Drug Deliv. 2017;24(1):1680-1689.

44. Sriraman SK, Salzano G, Sarisozen C, Torchilin V. Anti-cancer activity of doxorubicin-loaded liposomes co-modified with transferrin and folic acid. Eur J Pharm Biopharm. 2016;105:40-49.

45. Patil Y, Amitay Y, Ohana P, Shmeeda H, Gabizon A. Targeting of pegylated liposomal mitomycin-C prodrug to the folate receptor of cancer cells: Intracellular activation and enhanced cytotoxicity. $J$ Control Release. 2016;225:87-95.
International Journal of Nanomedicine

\section{Publish your work in this journal}

The International Journal of Nanomedicine is an international, peerreviewed journal focusing on the application of nanotechnology in diagnostics, therapeutics, and drug delivery systems throughout the biomedical field. This journal is indexed on PubMed Central, MedLine, CAS, SciSearch ${ }^{\circledR}$, Current Contents ${ }^{\circledR} /$ Clinical Medicine,

\section{Dovepress}

Journal Citation Reports/Science Edition, EMBase, Scopus and the Elsevier Bibliographic databases. The manuscript management system is completely online and includes a very quick and fair peer-review system, which is all easy to use. Visit http://www.dovepress.com/ testimonials.php to read real quotes from published authors. 